\title{
Strukturwandel fordert Neuausrichtung von Geschäftsmodellen
}

\author{
Erst wenn das Funktionieren vieler \\ Institutionen, Strukturen und Subsysteme vom \\ Wachstumsparadigma abgekoppelt wird und \\ Alternativen erkennbar sind, wird die Politik von \\ ihrer Wachstumsfixierung abrücken. \\ Von Irmi Seidl und Angelika Zahrnt
}

bleibt oder gering ausfällt (Seidl / Zahrnt 2010). Erst wenn das Funktionieren dieser Systeme vom Wachstumsparadigma abgekoppelt wird und Alternativen erkennbar sind, wird die Politik von ihrer Wachstumsfixierung abrücken.

Auch viele Unternehmen sind auf Wirtschaftswachstum angewiesen, treiben es durch ihre Strategien an, sind aber auch aufgrund von Rahmenbedingungen und eigener Unternehmensverfassung dazu gezwungen. Doch nicht alle Unternehmen sind gleich stark auf Wachstum ausgerichtet. Bakker et al. bestätigten 1999 mit einer empirischen Befragung, dass große Unternehmen stärker wachstumsorientiert sind als kleine. Binswanger (2006) geht davon aus, dass Unternehmen grundsätzlich einem Wachstumszwang unterliegen, da während der Produktionsphase bzw. vor dem Verkauf der Produkte der Einsatz von Eigen- und Fremdkapital notwendig ist, der verzinst werden muss. Die Zinsen können nur durch zusätzliche Produktion erwirtschaftet werden. Kapitalintensive Unternehmen unterliegen einem stärkeren Wachstumszwang als wenig kapitalintensive. Hinzu kommt nach Binswanger der Wachstumsdrang, der sich unter anderem durch Unternehmensziele, Wettbewerbssituation, Branche, Größe und Ort des Marktes und Erwartung von Kursgewinnen ergibt. Insbesondere börsennotierte Aktiengesellschaften unterliegen sowohl einem Wachstumszwang wie auch -drang - und dies deutlich mehr als Personengesellschaften, Stiftungen oder Genossenschaften. Auch steuerliche Anreize treiben Unternehmenswachstum an. In der Literatur gibt es eine Vielzahl von Vorschlägen, wie Steuern so gestaltet werden können, dass sie (Unternehmens-)Wachstum fördern (zum Beispiel Keuschnigg 2005; OECD 2009). Eine kritische Diskussion hierzu gibt es bisher nicht.

Eine Ablösung der Wachstumsorientierung von Unternehmen und die Entwicklung von Wachstumsneutralität drängt sich aus zwei Gründen auf: Zum einen löst Wirtschaftswachstum kaum mehr Probleme (zum Beispiel Arbeitsmarkt, sozialer Ausgleich), schafft allerdings viele (zum Beispiel Umwelt, Krisenanfälligkeit, Staatsverschuldung) (Jackson 2009, Miegel 2010; Seidl / Zahrnt 2010). Zum anderen sind die Wachstumsraten in den reichen westlichen Ländern seit Jahrzehnten rückläufig und werden sich in den nächsten Jahren auf sehr niedrigem Niveau bewegen. Folglich werden Absatzmärkte in den Industrieländern stagnieren und damit auch das unternehmerische Wachstum. Unternehmen müssen sich so aufstellen, dass sie vom Wachstum unabhängig dauerhaft ihre Existenz sichern können.

Neben diesen externen Zwängen als Auslöser kann Wachstumsneutralität auch in Phasen von Wirtschaftswachstum $\rightarrow$ Unternehmen) in existenzielle Gefahr, wenn Wachstum aus- 


\section{"Auch Orientierung an langfristigem Bestand, Qualität und Mitarbeiter(innen)zufriedenheit kann wirtschaftlich erfolgreich sein."}

eine mögliche unternehmerische Strategie sein. In der Tat gibt es durchaus wachstumsneutrale Unternehmen - in der Regel kleine oder mittelständige Unternehmen. Sie verdeutlichen, dass Unternehmen nicht zwangsläufig wachsen müssen und dass die prioritäre Orientierung an anderen Zielen - wie langfristiger Bestand, Qualität, Mitarbeiter(innen)zufriedenheit - wirtschaftlich erfolgreich sein kann. Mit einer solchen Ausrichtung sind diese Unternehmen als sogenannte Postwachstumspioniere gut vorbereitet auf eine mögliche bis wahrscheinliche Zukunft ohne steigende gesamtwirtschaftliche Produktion. Gleichwohl: Eine Postwachstumsgesellschaft wird auch für diese Unternehmen eine Umstellung und Herausforderung bedeuten.

Bevor wir diese Herausforderungen diskutieren, stellt sich die Frage, wie eine solche Postwachstumsgesellschaft aussehen dürfte. Zunächst eine Definition einer Postwachstumsgesellschaft: Diese ist nicht existentiell auf Wirtschaftswachstum angewiesen, es findet keine Politik zur Erhöhung des Wirtschaftswachstums statt, bislang wachstumsabhängige Subsysteme werden umgebaut und der Energie- und Ressourcenverbrauch werden entsprechend den Nachhaltigkeitszielen heruntergefahren. Somit gibt es in einer Postwachstumsgesellschaft weder ein Wachstumsgebot noch ein Wachstumsverbot.

Bei der Beschreibung, wie eine Postwachstumsgesellschaft konkret aussehen könnte, sind auch künftige Trends einzubeziehen. Dies sind rückläufige Wachstumsraten und damit Unternehmensgewinne, deutlich steigende Energie- und Ressourcenpreise und damit höhere Lebenshaltungskosten, we niger Erwerbsarbeit und in der Folge mehr Eigen- und Versor gungsarbeit, gemeinsam genutzte Güter und Dienstleistungen, demografischer Wandel und ein aus diesen Faktoren resultierender Strukturwandel. Unternehmen werden gefordert sein, ihre Geschäftsmodelle auf ein solches verändertes Umfeld auszurichten.

\section{Unternehmerische Herausforderungen}

Im Bereich der Produkte und Dienstleistungen wird die Herausforderung darin liegen, diese zusammen und dabei in Funktionen und Systemen zu denken. Sind bisher die Rollen von Produzent(inn)en und Konsument(inn)en weitgehend getrennt, dürften sich verschiedene Formen von Ko-Produktionen entwickeln - zum Beispiel bei der Produktentwicklung oder in Form von „Mit“-Produktion. Ein erstes Beispiel ist der Lebensmittelhandel, bei dem sich die Kooperation mit Bauern (Bäuerinnen) und Kund(inn)en anbietet: Letztere könnten an der Erzeugung von Lebensmitteln beteiligt werden, indem sie sich beispielsweise an Feld- oder Erntearbeit beteiligen. Ein zweites Beispiel sind Industriegüter und -dienstleistungen: Ähnlich wie bei Computersoftware, die oftmals unter Beteiligung vieler externer Nutzer(innen) und Entwickler(innen) (weiter-)entwickelt wird, ist auch denkbar, dass Mit-Entwicklung und Mit-Produktion auch bei materiellen Produkten und Dienstleistungen stattfindet. Beteiligte der sogenannten Repair-Revolution könnten so zum Beispiel Qualitätsverbesserungen vorantreiben. Ein anderes neues Geschäftsfeld ist die Sparberatung: So wie sich in den vergangenen Jahren Energieagenturen als Energiesparberatungsunternehmen etablieren konnten, könnte sich Sparberatung auch auf Ressourcen, Mobilität und Konsumgüter ausdehnen. Weiter: Während heute Unternehmen vor allem Konsumgüter für den individuellen Besitz und Verbrauch herstellen, könnte in Zukunft die Eignung von Gütern für den gemeinschaftlichen Besitz ein wichtiges Kriterium werden. Bei Dienstleistungen dürfte es dann um den gemeinschaftlichen Besitz oder eine gemeinschaftliche Nutzung gehen (Beispiel Car-Sharing). Neue unternehmerische Handlungsfelder dürften auch durch eine verstärkte Nachfrage nach Gemeingütern wie zum Beispiel gemeinschaftlichen Wohn-, Garten- oder Kulturprojekten in kommunaler oder privater Verantwortung entstehen. Ebenfalls ein neues Geschäftsfeld dürfte der möglicherweise steigende Anteil der Eigenversorgung werden: Produkte und Dienstleistungen könnten angeboten werden, die diese Aktivitäten im häuslichen, gärtnerischen, handwerklichen Bereich ermöglichen und durch Bildungsangebote die nötigen Kenntnisse vermitteln. Schließlich bietet die Produktqualität Profilierungsmöglichkeiten: Der Trend zu Kurzlebigkeit aufgrund schlechter Qualität (Geplante Obsoleszenz) wird von Kund(inn)en zunehmend kritisiert und ist aufgrund knapper werdender Ressourcen ökologisch unhaltbar. Die angedeuteten Möglichkeiten der Umorientierung oder Erweiterung der Produktund Dienstleistungspalette erfordern in Unternehmen neue Qualifikationen, insbesondere auch eine größere Offenheit für die Bedürfnisse von Kund(inn)en und Partner(inne)n sowie Kommunikationsbereitschaft und -fähigkeit.

Eine besondere Herausforderung ist das sinkende gesamtgesellschaftliche Arbeitsvolumen; Staat, Gesellschaft, Unternehmen und insbesondere Personalabteilungen und Arbeits- und Projektplaner werden gleichermaßen gefordert sein, unterschiedlichste Arbeitszeitmodelle zu entwickeln und zu implementieren. Weiter dürften in einer Postwachstumsgesellschaft qualitative Faktoren am Arbeitsplatz wichtiger werden - unter anderem wegen des demografischen Wandels und einem möglichen Bedeutungsverlusts von Karriere. Schon jetzt gewinnen für Arbeitnehmer(innen) andere Lebensaspekte als Erwerbsarbeit an Bedeutung. Insbesondere die individuelle Work-Life-Ba- 
lance und die Verbindung von Familie und Beruf wird Unternehmen zunehmende Flexibilität, Kreativität und Kompetenz in der Planung und Mitarbeiter(innen)führung abverlangen.

Im Hinblick auf die räumlichen Produktions- und Kund(inn)enbeziehungen dürften sich die Gewichte zwischen Globalisierung und Regionalisierung verändern. Steigende Treibstoff- und Rohstoffkosten dürften den räumlich nahen Bezug von Rohstoffen und Vorprodukten fördern und Unternehmenskooperationen zugunsten von Einsparung und Wiederverwendung anregen - so zum Beispiel in der Abwärme- und Kaskadennutzung oder der Produktweiternutzung über Tausch- und Handelsbörsen (zum Beispiel von Bauteilen). Die Rohstoffgewinnung aus Altstoffen dürfte neue Geschäftsfelder im Bereich von Urban Mining und Dekonstruktion eröffnen und damit auch zugleich die Konstruktion neuer Gebäude und Produkte beeinflussen. Weiter wird die Organisation und Betreuung derartiger kommunaler und regionaler Netzwerke neue Beratungsfelder eröffnen. Insgesamt ist zu erwarten, dass eine verstärkte Dienstleistungsorientierung zu einer stärkeren Nähe zu Kund(inn)en führt. Parallel zu den stagnierenden Märkten in Europa und dem beginnenden Strukturwandel verlagern manche Unternehmen ihre Aktivitäten in wachsende Märkte in Schwellen- und Entwicklungsländern oder bauen dort ein zweites Standbein auf. Daraus könnten sich die Risiken der Abhängigkeit von Exportmärkten verstärken, und in absehbarer Zeit dürften auch dort die Wachstumsraten zurückgehen.

Was künftige Unternehmen anbelangt, dürften weniger wachstumsorientierte Formen wie Genossenschaften, Stiftungen, Social Entrepreneurs oder traditionelle klein- und mittelständische Unternehmen an Bedeutung gewinnen, weil auf ihnen ein geringerer Wachstumsdruck liegt. Hier dürften gerade für genossenschaftlich organisierte Banken neue Beratungsaufgaben entstehen. Der erkennbare Zuwachs an Genossenschaften vor allem im Energiebereich verweist bereits auf Beratungsbedarf.

\section{Gesellschaftspolitischer Beitrag von Unternehmen}

Unternehmen sind gefordert, sich für veränderte Rahmenbedingungen einzusetzen und sich in die gesellschaftliche und politische Debatte einzumischen, um die Transformation zu einer Postwachstumsgesellschaft zu unterstützen und so die natürlichen Lebensgrundlagen zu erhalten und die sozialen Unterschiede zu verringern. Konkret geht es um Rahmenbedingungen wie die Internalisierung externer Kosten durch Energie- und Ressourcensteuern, Cap-Systeme oder den Emissionshandel, die zugleich für Unternehmen gleiche Voraussetzungen schaffen. Damit einher geht die Forderung nach einer Streichung umweltschädlicher Subventionen. Weiter ist Engagement für international bessere Standards im Umwelt- und im Sozialbereich notwendig - zunächst, da dies erfahrungsgemäß eine langwierige Aufgabe ist, über Branchenabkommen.
Ein weiterer Beitrag ist das Sichtbarmachen von ökologischen und sozialen externen Kosten durch eine eigene Rechnungslegung (von integrierter Berichterstattung bis zu Gemeinwohlbilanzen), was zugleich eigene Handlungsspielräume eröffnet und die Notwendigkeit verbindlicher Rahmensetzungen deutlich macht. Unternehmen können damit im Wettbewerb die eigene Verantwortung glaubhaft dokumentieren. $\mathrm{Zu}$ verbesserten Rahmenbedingungen für nachhaltiges Wirtschaften gehört auch, über Regelungen zu Haftung, Garantien und verbindlichen Standards die Qualität von Produkten zu erhöhen.

Viele der Herausforderungen, denen sich Unternehmen in einer Postwachstumsgesellschaft stellen müssen, zeichnen sich heute schon ab. Antworten werden bereits erprobt. Eine umfassende Auseinandersetzung mit der Frage, wie sich Unternehmen in einer Postwachstumsgesellschaft verändern und entwickeln können, steht aber noch aus. Klar ist, dass es auch in einer Postwachstumsgesellschaft Dynamik und Strukturwandel geben wird. Das heißt, Unternehmen werden wachsen, schrumpfen und ohne Wachstum erfolgreich sein. Solche wachstumsneutralen Unternehmen können mit ihren eigenen Erfahrungen eine Vorreiterrolle als Postwachstumspioniere spielen.

\section{Literatur}

Binswanger, H.-C. (2006): Die Wachstumsspirale. Geld, Energie und Imagination in der Dynamik des Marktprozesses. Marburg, Metropolis-Verlag.

Hicks, J.R. (1966): Growth and Anti-Growth. In: Oxford Economic Papers 18, 3/1966. S. 257-269.

Jackson, T. (2009): Prosperity without Growth. The Transition to a Sustainable Economy. London, Sustainable Development Commission.

Johansson, Å. et al. (2008): Tax and Economic Growth. Working Paper 620, OECD Economics Department.

Keuschnigg, C. (2004): Eine Steuerreform für mehr Wachstum in der Schweiz. Zürich, Avenir Suisse.

Miegel, M. (2010): Exit. Wohlstand ohne Wachstum. Berlin, Propyläen-Verlag. Seidl, I. / Zahrnt, A. (Hrsg.) (2010): Postwachstumsgesellschaft. Konzepte für die Zukunft. Marburg, Metropolis-Verlag.

Ulrich, H. (1984): Management. Bern/Stuttgart/Wien, Haupt.

\section{AUTORINNEN + KONTAKT}

Irmi Seidl ist Leiterin der Forschungseinheit Wirtschafts- und Sozialwissenschaften der Eidg. Forschungsanstalt WSL.

Angelika Zahrnt ist Ehrenvorsitzende des BUND und Mitglied im Rat für Nachhaltige Entwicklung.

Irmi Seidl, Eidg. Forschungsanstalt WSL, Zürcherstr. 111, 8903 Birmensdorf, Schweiz, Tel.: +41 447392 324,

E-Mail: irmi.seidl@wsl.ch Angelika Zahrnt, Hollmuthstr. 2a, 69151 Neckargemünd, Tel.: +496223 72226, E-Mail: angelika.zahrnt@bund.net
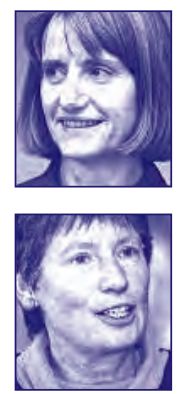
Copyright (C) 2013, IÖW und oekom Verlag. Die Nutzung des Artikels ist Abonnenten von Ökologisches Wirtschaften vorbehalten. Nachdruck und Vervielfältigung des Artikels einschließlich Speicherung und Nutzung auf optischen und elektronischen Datenträgern nur mit Zustimmung der Redaktion von Ökologisches Wirtschaften (http://www.oekologischeswirtschaften.de). 